\title{
EVALUATION OF SOME CUCURBITACEOUS ROOTSTOCKS 1- FOR RESISTANCE / SUSCEPTIBILITY TO ROOT- KNOT NEMATODE AND FUSARIUM WILT UNDER SCREEN-HOUSE CONDITIONS
}

\author{
AMIN, A.W. ${ }^{1}$, M. ABD-EL WANIS ${ }^{2}$ and TOMADER G. ABDEL RAHMAN ${ }^{3}$ \\ 1. Cairo University, Faculty of Agriculture, Zoology \& Nematology Department, \\ 2. Protected Cultivation Dept. Horticulture Research Institute ARC, \\ 3. Institute of Vegetable, Plant Pathology Dept., ARC,
}

(Manuscript received 3 November 2011)

\begin{abstract}
Southern root-knot nematode, Meloidogyne incognita and Fusarium wilt, Fusarium oxysporum are the most serious soil borne diseases in soil of cucumber. The present study aimed to evaluate some cucurbit rootstocks for their resistance and/or susceptibility to root-knot nematode and fusarium wilt fungus in two successive seasons (2009 and 2010) under screen-house conditions. Data indicated that in autumn season, winter squash ( Cucurbita maxima) was highly resistant and resistant when the pots were inoculated with $M$. incognita only or nematode plus fungus, respectively. Lagenaria sicenaria rootstock was moderately resistant in both cases of inoculation. Other rootstocks were susceptible to nematode or nematode plus fungus except Luffa aegyptiaca, as it was highly susceptible to nematode. While, in spring season, Benincasa hispida and hybrid 6001 were moderately resistant to infection by the root-knot nematode. Other rootstocks were susceptible to nematode or nematode plus fungus except Cucurbita moschata, Luffa acutangula, and Cucumis sativus var.Hesham, as they were highly susceptible to $M$. incognita only. Also, Lagenaria siceraria, Luffa acutangula and Cucumis sativus var. Hesham were highly susceptible to nematode and fungus. The results obtained on the enzyme contents showed that some cucurbit rootstocks exhibited the highest contents of polyphenol oxidase, peroxidase and lignin contents being the lowest in some rootstocks. Significant and highly significant reductions in shoot and root fresh weights, root and shoot lengths were recorded in the most cucurbit rootstocks in autumn and spring seasons as a result of nematode, nematode plus fungus or fungus infections compared to non- infected rootstocks.
\end{abstract}

Keyword: Cucurbit rootstocks, Fusarium wilt, Root-knot nematode, soil-borne diseases.

\section{INTRODUCTION}

As for in vegetable crops production in greenhouses, soil-borne diseases and nematodes cause most of the damage from continuous cropping (Oda, 1999). Cucumber crop suffers from several infections by serious nematodes and fungal diseases. Southern root-knot nematode, Meloidogyne incognita Chitwoodi and fusarium wilt fungus, Fusarium oxysporum are the most serious soil borne diseases in 
cucumber rhizosphere (Sharma et al (1995). Owens and Novothy (1966) found that plant tissues infested with nematodes showed a considerable increase in the levels of free amino acids, organic acids and protein.

There are several ways to control the nematodes and fusarium wilt, none of them proved to be good and effective. No commercial cultivars or hybrids of cucumber are recorded as resistant to nematode diseases. Therefore, the only possible approach to overcome such diseases is using grafting of resistant rootstocks as an active, safe and clean method. Grafting onto resistant rootstocks of wild or domesticated species of cucurbit plant is the most known means to protect the scion from the attack by these diseases. Trionfetti et. al. (2002) mentioned that rootstock influence on disease resistance as well as productivity and quality of scion fruit is important in determining the potential utility of grafting applications. Zhang et. al. (2010) concluded that phenylpropane metabolites and key enzymes had close correlation with resistance to southern root-knot nematode in grafting cucumber seedling.

This work was planned to study the resistance and /or susceptibility of cucumber and some wild and domesticated cucurbit rootstocks to infection with rootknot, M. incognita nematode and Fusarium oxysporum as a part of our sequence study to evaluate cucumber plants grafted onto some cucurbitaceous plants to avoid infection with root-knot nematode and fusarium wilt diseases.

\section{MATERIALS AND METHODS}

The fungus and nematode were isolated from diseased cucumber plants collected from the inspected fields and from the greenhouses located at Kaha Research Station, Qaluobiya governorate Horticultural Research Institute, Agricultural Research center. Cucumber plants showing the typical symptoms of wilt and galls of nematodes were collected during 2009-2010. The plant samples were uprooted and put in plastic bags which were kept in a cool container during transportation to the laboratory. The samples were kept in a refrigerator at $5 \mathrm{c} 0$ for further studies. The fungus was isolated on Potato Dextrose Agar (PDA). The inoculums used in the foregoing studies consisted of uniform agar discs of $4 \mathrm{~mm}$. in diameter bearing 7 days old growth of the desired fungus under aseptic conditions. The inoculum was grown in $500 \mathrm{ml}$. milk bottles containing the following sterilized substrate per bottle: Washed dried maize $75 \mathrm{gm}$, Washed dried coarse sand $100 \mathrm{gm}$ and tap water $65 \mathrm{ml}$.The bottles were autoclaved before being incubated at $25 \mathrm{C} \circ$ for two weeks to obtain sufficient growth of the fungus. The inoculum was then thoroughly mixed with the soil sterilized 
at the desired rate and time. The level of infection as affected by the level of inoculums was $5 \mathrm{gm}$ per kilogram for both sterilized and unsterelized growing media were used.

Thirteen cucurbitaceous rootstocks seeds (Table 1) were sown in $25 \mathrm{~cm}-\mathrm{d}$ plastic pots filled with $1 \mathrm{~kg}$ sandy-loamy soil $(1: 1 \mathrm{v} / \mathrm{v})$ free of plant fungus and parasitic nematodes.

\section{Source of the tested plants}

Seeds of the tested plants were obtained from various sources as mentioned in Table (1).

Table 1. Source of rootstocks evaluated against $F$. oxysporum and M. incognita isolated from infested cucumber plants.

\begin{tabular}{|c|c|c|}
\hline Rootstocks & Scientific name & Source \\
\hline Bottle gourd & $\begin{array}{c}\text { Lagenaria sicenaria var. } \\
\text { calabsha }\end{array}$ & Pakistan \\
\hline Bottle gourd & Lagenaria sicenaria & $\begin{array}{c}\text { Horticulture Research,Institute, } \\
\text { Egypt }\end{array}$ \\
\hline $\begin{array}{c}\text { Butter nut squash (winter } \\
\text { squash) }\end{array}$ & $\begin{array}{c}\text { Cucurbita maxima }{ }^{*} \text { Cucrbita } \\
\text { moshata }\end{array}$ & $\begin{array}{c}\text { Horticulture Research,Institute, } \\
\text { Egypt }\end{array}$ \\
\hline Cucurbita maxima Varity & Cucurbita maxima & Pakistan \\
\hline Cucumber & Cucumis sativus var. Hesham & Royal Sluis Comp.Holland \\
\hline Fig leaf gourd & Cucurbita ficifolia & Wild seeds \\
\hline Hercules gourd & Lagenaria longissima & $\begin{array}{c}\text { Horticulture Research, Institute, } \\
\text { Egypt }\end{array}$ \\
\hline Hybrid Arcola & $\begin{array}{c}\text { C.maxima } x \text { C.moshata F1 } \\
\text { No.6001 }\end{array}$ & Nunhems Comp. \\
\hline Hybrid Charmatoza & Cucurbita maxima & Taky seeds Comp. \\
\hline Luffa black seed & Luffa aegyptiaca & $\begin{array}{c}\text { Horticulture Research, Institute, } \\
\text { Egypt }\end{array}$ \\
\hline Luffa white seed & Luffa acutangula & Pakistan \\
\hline Pumpkin & Cucurbita moschata & $\begin{array}{c}\text { Horticulture Research, Institute, } \\
\text { Egypt }\end{array}$ \\
\hline
\end{tabular}


Half number of pots was infested with 500 infective stages of Meloidogyne incognita (obtained from egg massing

by incubating in water) around the seedling roots. The pots were divided into four groups. The first group was inoculated with root-knot nematode, Meloidogyne incognita only and the second group received nematodes and Fusarium oxysporum around the seedling roots, the third group received of infested soil by Fusarium oxysporum only and the fourth group served as control plant (uninoculated).

There were five replicates for each treatment and the pots were arranged in a completely randomized design in greenhouse. The pots were watered as needed. After nine weeks of nematodes and fungus inoculations, roots of plants were carefully uprooted and nematodes in soil and roots were counted and recorded as root gall and egg-mass indices according to Taylor and Sasser (1978). Disease incidence caused by Fusarium oxysporum during the growth period was recorded. The number of galls, females and egg-masses and reproductive factor were calculated for root-knot nematodes as compared to uninoculated pots calculated for root-knot nematodes. Length and weight of shoots and roots were recorded at the end of autumn 2009 and spring 2010. The aforementioned characters were investigated in the presence of nematodes and/or wilt fungus diseases compared to control plants. This experiment Was repeated twice in autumn 2009 and spring 2010 with the tested cucurbit rootstocks. Data were statistically analyzed using new Least Significant Difference (New LSD) (Waller and Duncan, 1969).

\section{Chemical Analysis (Oxidative - reductive enzyme activities) and lignin content}

Roots of diseased plants were harvested at spring and autumn seasons after inoculation with Fusarium and nematodes by cutting them at the root system for determining the activity of peroxidase and polyphenoloxidase and lignin content. Enzyme activities were determined in the roots of inoculated plants as recommended by Maxwell and Batman (1967). Peroxidas assay was determined according to the methods described by Allam and Hollis (1972) and lignin content was determined according to by Anonymous (1951). 


\section{RESULTS}

\section{1- Susceptibility of some cucurbit plant species grown in soil infected by Meloidogyne incognita, and / or Fusarium oxysporum}

Most of the root system of the cucurbit rootstocks grown in soil infected by $M$. incognita was uniformly galled, where the gall/egg-masses index ranged from 1 to 5 in autumn season and from 3 to 5 in spring season (Tables 2, 4 and Figs. 1, 2).

In autumn season, data presented in Table (2) and Fig (1) indicates that the cucurbit rootstocks varied in their rates of susceptibility according to Taylor and Sasser 1978. It was found that Cucurbita maxima were highly resistant and resistant to M.incognita only or nematode plus fungus, respectively. Lagenaria siceraria was moderately resistant to root-knot nematode against nematode or nematode plus fungus. Other Cucurbit rootstocks were susceptible (S) to nematode or nematode plus fungus except rootstocks of Luffa aegyptiaca was highly susceptible to nematode only. When Cucurbit stocks, in autumn, were infected by fungus, F.oxysporum, all of then were highly resistant (HS) to fungus except Cucumis sativus var. Hesham, as it was susceptible (S) to fungus. In spring season (Table 4), Benincasa hispida and cucurbita maxima ( hybrid 6001) were moderately resistant (MR) to infection by M.incognita only and M.incognita plus fusarium oxysporium. Other Cucurbit rootstocks were susceptible (S) to nematode and nematode plus fungus except Cucurbita mochata was highly susceptible to nematode only. Lagenaria sicenaria, Luffa acutangula and Cucumis sativus were highly susceptible to nematode plus fungus. When cucurbit rootstocks were inoculated with fungus only in spring, all of they were highly resistant to fungus except cucumis sativus var. Hesham, as it was susceptible to fungus. In Cucurbitaceous plants grown in soil infected with $M$. incognita only in autumn, the highest reproduction factor (RF) was 3.05 on Cucumis sativus var. Hesham followed by that (2.77) of the same plant variety when grown in soil infected with nematode plus fungus (Table 2) On the other hand, the highest RF in spring was 2.64 when Cucumis sativus var.Hesham was grown in soil infected with M.incognita only followed by RF (3.12) when the same plant species was grown in soil infected with nematode plus fungus (Table 4 and Fig (2)).

\section{2- Cucurbit fresh plant criteria}

Significant and highly significant reduction in shoot and root fresh weights were recorded in most cucurbit plants in autumn and spring seasons (Tables 3and5). Severe significant and highly significant reduction were recorded in root and shoot 
length in both season on the cucurbit plants inoculated by $M$. incognita or/and $F$. oxysporum.

\section{3- Peroxidase and Polyphenoloxidase activities and lignin content of some cucurbit plants in relation to nematode and fungus infection}

Data presented in Table 6 indicate that Lagenaria sicenaria, cucurbita maxima $\mathrm{x}$ C. moschata (H.6001), Cucurbita maxima (Winter squash) and L. aegyptiaca showed the highest polyphenol oxidase content, being 0.059, 0.052, 0.052 and 0.47, respectively. Meanwhile, L.acutangula, C.maxima x C. moschata (H.6001) and Benincasa hispida showed the highest values of peroxidase content as the corresponding values were $1.053,0.985$ and 0.944 on the average, respectively. Concerning lignin content, it was found that C.maxima (Charmatoza), and L. sicenaria showed the highest values of lignin contents, being 0.419 and 0.378 , respectively in spring season. On the other hand, in autumn season L. sicenaria and Cucurbita maxima (Winter squash) showed the highest polyphenol oxidase content (0.065 and 0.063), respectively. Meanwhile, Luffa acutangula , Cucurbita maxima (Winter squash) and Cucurbita ficifolia showed the highest values of peroxides content. There corresponding values were $1.049,0.781$ and 0.722 on the average, respectively. Concerning lignin content, it was found that Lagenaria sicenaria and $\angle$ sicenaria var Calabsha showed the highest values of lignin contents, being (0.379 and 0.274 ), respectively. 
Table 2. Different parameters of Meloidogyne incognita or/and Fusarium oxysporum under Screen-house condition in autumn season.

\begin{tabular}{|c|c|c|c|c|c|c|c|c|c|c|}
\hline \multirow[b]{3}{*}{ Treatments } & \multirow[b]{3}{*}{$\begin{array}{c}\text { No. of } \\
\text { gall }\end{array}$} & \multicolumn{4}{|c|}{ Number of nematodes } & \multirow{3}{*}{ 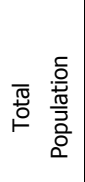 } & \multirow[b]{3}{*}{ RF* } & \multirow[b]{3}{*}{ GI/EI** } & \multirow{2}{*}{\multicolumn{2}{|c|}{ Fusarium wilt ${ }^{* * *}$}} \\
\hline & & \multirow[b]{2}{*}{ In soil } & \multicolumn{3}{|c|}{ In roots } & & & & & \\
\hline & & & $\begin{array}{l}\text { Dev. } \\
\text { stage }\end{array}$ & $\begin{array}{c}\text { Femal } \\
\text { es }\end{array}$ & $\begin{array}{c}\text { Egg } \\
\text { masses }\end{array}$ & & & & $\begin{array}{c}\% \text { of } \\
\text { disease }\end{array}$ & Index \\
\hline Cucurbita ficifolia (A) & 103.0 & 83.0 & 29.3 & 86.3 & 78.3 & 198.6 & 0.40 & $\begin{array}{c}5 / 4 \\
(\mathrm{HS})^{*}\end{array}$ & - & - \\
\hline Cucurbita ficifolia (B) & 48.0 & 53.0 & 16.0 & 40.0 & 40.0 & 113.0 & 0.22 & $4 / 4(S)$ & 92.0 & (HS) \\
\hline Cucurbita ficifolia (C) & - & - & - & - & - & - & - & - & 1.0 & (HR) \\
\hline Cucurbita moschata (Pumpkin) (A) & 49.3 & 53.0 & 24.6 & 32.3 & 30.7 & 109.9 & 0.22 & $4 / 4(S)$ & - & - \\
\hline Cucurbita moschata (Pumpkin) (B) & 49.3 & 52.0 & 27.3 & 37.3 & 35.3 & 116.6 & 0.23 & $4 / 4(S)$ & 1.0 & $(\mathrm{HR})$ \\
\hline Cucurbita moschata (Pumpkin) (C) & - & - & - & - & - & - & - & - & 1.0 & $(\mathrm{HR})$ \\
\hline $\begin{array}{l}\text { Cucurbita maxima (winter squash) } \\
\text { (A) }\end{array}$ & 2.0 & 59.6 & 3.6 & 2.3 & 1.6 & 63.5 & 0.13 & $1 / 1(\mathrm{HR})$ & - & - \\
\hline $\begin{array}{l}\text { Cucurbita maxima (winter squash) } \\
\text { (B) }\end{array}$ & 3.3 & 10.0 & 3.3 & 3.6 & 2.6 & 16.9 & 0.03 & $2 / 2(R)$ & 41.7 & $(\mathrm{R})$ \\
\hline $\begin{array}{l}\text { Cucurbita maxima (winter squash) } \\
\text { (C) }\end{array}$ & - & - & - & - & - & - & - & - & 28.3 & $(R)$ \\
\hline Lagenaria sicenaria (A) & 31.0 & 10.0 & 7.3 & 26.7 & 25.7 & 44.0 & 0.09 & $3 / 3(\mathrm{MR})$ & - & - \\
\hline Lagenaria sicenaria (B) & 28.3 & 51.7 & 8.6 & 23.3 & 23.3 & 83.5 & 0.17 & $3 / 3(\mathrm{MR})$ & 32.7 & $(R)$ \\
\hline Lagenaria sicenaria (C) & - & - & - & - & - & - & - & - & 1.0 & $(\mathrm{HR}$ \\
\hline $\begin{array}{l}\text { Lagenaria sicenaria var Calabsha } \\
\text { (A) }\end{array}$ & 68.7 & 25.0 & 14.0 & 58.6 & 57.3 & 97.6 & 0.20 & $4 / 4(S)$ & - & - \\
\hline $\begin{array}{l}\text { Lagenaria sicenaria var Calabsha } \\
\text { (B) }\end{array}$ & 35.0 & 10.0 & 14.0 & 49.0 & 28.0 & 72.0 & 0.15 & $4 / 4(S)$ & 62.3 & $(\mathrm{~S})$ \\
\hline $\begin{array}{l}\text { Lagenaria sicenaria var Calabsha } \\
\text { (C) }\end{array}$ & - & - & - & - & - & - & - & - & 10.7 & $(\mathrm{HR})$ \\
\hline Luffa acutangula (A) & 41.3 & 10.0 & 23.6 & 39.3 & 31.6 & 72.9 & 0.15 & $4 / 4(S)$ & - & - \\
\hline Luffa acutangula (b) & 44.3 & 10.0 & 24.6 & 76.6 & 47.6 & 74.9 & 0.15 & $4 / 4(S)$ & 95.3 & (HS) \\
\hline Luffa acutangula (C) & - & - & - & - & - & - & - & - & 34.0 & $(\mathrm{R})$ \\
\hline Luffa aegyptiaca (A) & 124.6 & 54.0 & 46.3 & 91.6 & 87.3 & 191.9 & 0.38 & $5 / 4(\mathrm{HS})$ & - & - \\
\hline Luffa aegyptiaca (B) & 72.6 & 10.0 & 26.7 & 66.6 & 56.6 & 103.2 & 0.21 & $4 / 4(S)$ & 61.0 & (S) \\
\hline Luffa aegyptiaca (C) & - & - & - & - & - & - & - & - & 1.0 & $(\mathrm{HR})$ \\
\hline Lagenaria longissima (A) & 75.3 & 10.0 & 30.0 & 74.0 & 73.0 & 114.0 & 0.22 & $4 / 4(S)$ & - & - \\
\hline Lagenaria longissima (B) & 96.3 & 382.6 & 26.6 & 85.3 & 79.3 & 494.5 & 0.99 & $4 / 4(S)$ & 1.0 & $(\mathrm{HR})$ \\
\hline Lagenaria longissima (C) & - & - & - & - & - & - & - & - & 1.0 & $(\mathrm{HR})$ \\
\hline Cucumis sativus var. Hesham (A) & 54.0 & 1381.6 & 53.3 & 90.0 & 45.6 & 1524.9 & 3.05 & $4 / 4(S)$ & - & - \\
\hline Cucumis sativus var. Hesham (B) & 75.6 & 1293.3 & 13.3 & 77.0 & 72.0 & 1383.6 & 2.77 & $4 / 4(S)$ & 82.33 & (HS) \\
\hline Cucumis sativus var. Hesham (C) & - & - & - & - & - & - & - & - & 58.3 & $(\mathrm{~S})$ \\
\hline New L.S.D. (5\%) & 33.1 & 7.88 & 11.12 & 27.2 & 32.7 & - & - & - & - & - \\
\hline
\end{tabular}

A $=$ Nematodes Infected Plants, B = Nematodes and fungi infected plants, C= fungi infected plants Scales expressed by letter(S) in parenthesis are plant response to M. incognita infected according to Taylor and Sasser (1978)

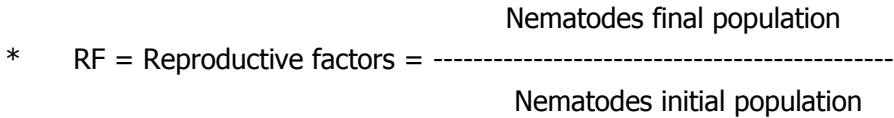

** $\quad$ GI/EI $=$ galls and egg-masses index according to Taylor and Sasser 1978

***Disease symptoms index according to Kesevan and Chounhury, (1977),

(0)= Immune (1), (1)= Highly Resistant (HR), (2)= Resistant (R), (3)= Susceptible (S), (4)= Highly Susceptible (HS). 
1568 EVALUATION OF SOME CUCURBITACEOUS ROOTSTOCKS ${ }^{1-}$ FOR RESISTANCE / SUSCEPTIBILITY TO ROOT- KNOT NEMATODE AND FUSARIUM WILT UNDER SCREEN-HOUSE CONDITIONS

Table 3. Different parameters of some cucurbit rootstocks to infected with Meloidogyne incognita or/and Fusarium oxysporum under Screen-house condition in autumn season.

\begin{tabular}{|c|c|c|c|c|}
\hline Treatments & $\begin{array}{l}\text { Root } \\
\text { Length } \\
(\mathrm{cm})\end{array}$ & $\begin{array}{c}\text { Root } \\
\text { weight } \\
(\mathrm{g})\end{array}$ & $\begin{array}{l}\text { Shoot } \\
\text { Length } \\
(\mathrm{cm})\end{array}$ & $\begin{array}{c}\text { Shoot } \\
\text { weight } \\
(\mathrm{g})\end{array}$ \\
\hline Cucurbita ficifolia (A) & 26.00 & 4.93 & 77.76 & 18.53 \\
\hline Cucurbita ficifolia (B) & 19.26 & 2.40 & 54.80 & 9.60 \\
\hline Cucurbita ficifolia (C) & 26.00 & 4.93 & 77.76 & 18.53 \\
\hline Cucurbita ficifolia (D) & 24.66 & 5.40 & 170.33 & 17.1 \\
\hline Cucurbita moschata (Pumpkin) (A) & 7.66 & 3.63 & 31.70 & 11.33 \\
\hline Cucurbita moschata (Pumpkin) (B) & 12.50 & 2.30 & 47.66 & 15.07 \\
\hline Cucurbita moschata (Pumpkin) (C) & 12.50 & 3.63 & 44.33 & 14.83 \\
\hline Cucurbita moschata (Pumpkin) (D) & 21.00 & 5.10 & 135.00 & 16.66 \\
\hline Cucurbita maxima (winter squash) (A) & 29.56 & 9.96 & 69.16 & 7.16 \\
\hline Cucurbita maxima (winter squash) (B) & 19.70 & 6.73 & 50.83 & 6.00 \\
\hline Cucurbita maxima (winter squash) (C) & 21.33 & 3.73 & 21.13 & 13.40 \\
\hline Cucurbita maxima (winter squash) (D) & 33.66 & 10.86 & 131.66 & 10.16 \\
\hline Lagenaria sicenaria $(\mathrm{A})$ & 9.33 & 3.90 & 70.96 & 12.17 \\
\hline Lagenaria sicenaria (B) & 20.66 & 1.95 & 66.00 & 13.70 \\
\hline Lagenaria sicenaria (C) & 20.66 & 3.90 & 66.00 & 13.68 \\
\hline Lagenaria sicenaria (D) & 18.33 & 4.46 & 137.66 & 17.10 \\
\hline Lagenaria sicenaria var Calabsha (A) & 23.00 & 4.90 & 82.66 & 23.73 \\
\hline Lagenaria sicenaria var Calabsha (B) & 12.16 & 3.16 & 64.40 & 19.03 \\
\hline Lagenaria sicenaria var Calabsha (C) & 23.00 & 4.90 & 82.66 & 23.73 \\
\hline Lagenaria sicenaria var Calabsha (D) & 35.33 & 7.87 & 147.33 & 15.66 \\
\hline Luffa acutangula (A) & 23.00 & 6.53 & 79.16 & 9.80 \\
\hline Luffa acutangula (B) & 23.46 & 2.40 & 70.80 & 7.66 \\
\hline Luffa acutangula (C) & 17.00 & 2.66 & 36.00 & 9.70 \\
\hline Luffa acutangula (D) & 24.33 & 5.60 & 163.66 & 11.86 \\
\hline Luffa aegyptiaca (A) & 21.33 & 8.16 & 80.33 & 11.63 \\
\hline Luffa aegyptiaca (B) & 19.06 & 3.90 & 50.36 & 8.00 \\
\hline Luffa aegyptiaca (C) & 21.50 & 8.13 & 80.50 & 11.66 \\
\hline Luffa aegyptiaca (D) & 25.33 & 14.00 & 154.00 & 18.00 \\
\hline Lagenaria longissima (A) & 23.50 & 4.00 & 26.00 & 9.80 \\
\hline Lagenaria longissima (B) & 18.66 & 1.73 & 24.16 & 7.33 \\
\hline Lagenaria longissima (C) & 27.76 & 3.16 & 26.00 & 11.53 \\
\hline Lagenaria longissima (D) & 22.00 & 6.90 & 140.33 & 14.40 \\
\hline Cucumis sativus var. Hesham (A) & 19.26 & 3.20 & 67.96 & 4.10 \\
\hline Cucumis sativus var. Hesham (B) & 21.13 & 2.36 & 36.86 & 3.76 \\
\hline Cucumis sativus var. Hesham (C) & 18.33 & 1.63 & 56.00 & 4.20 \\
\hline Cucumis sativus var. Hesham (D) & 27.33 & 3.10 & 162.00 & 4.86 \\
\hline New L.S.D. (5\%) & 10.10 & 3.10 & 33.50 & 5.90 \\
\hline
\end{tabular}

$A=$ Nematodes Infected plants

$\mathrm{C}=$ Fusarium infected plants

$B=$ Nematodes and fungi infected plants

$\mathrm{D}=$ Non - infected plants (control) 
Table 4. Different parameters of Meloidogyne incognita or/and Fusarium oxysporum under Screen-house condition in spring season.

\begin{tabular}{|c|c|c|c|c|c|c|c|c|c|c|}
\hline \multirow{3}{*}{ Treatments } & \multirow{3}{*}{$\begin{array}{l}\text { No. of } \\
\text { gall }\end{array}$} & \multicolumn{4}{|c|}{ Number of nematodes } & \multirow{3}{*}{ 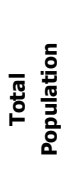 } & \multirow[b]{3}{*}{ RF* } & \multirow[b]{3}{*}{ GI/EI** } & \multirow{2}{*}{\multicolumn{2}{|c|}{ Fusarium wilt }} \\
\hline & & \multirow[b]{2}{*}{ In soil } & \multicolumn{3}{|c|}{ In roots } & & & & & \\
\hline & & & $\begin{array}{l}\text { Dev. } \\
\text { stage }\end{array}$ & Females & $\begin{array}{c}\text { Egg } \\
\text { masses }\end{array}$ & & & & $\begin{array}{c}\% \text { of } \\
\text { disease } \\
* * *\end{array}$ & Index \\
\hline Cucurbita ficifolia (A) & 90.7 & 90.3 & 28.7 & 66.0 & 36.0 & 185.0 & 0.37 & $4 / 4(\mathrm{~S})^{*}$ & - & - \\
\hline Cucurbita ficifolia (B) & 52.0 & 56.3 & 15.0 & 38.7 & 30.0 & 110.0 & 0.22 & 4/4 (S) & 25.0 & (HR) \\
\hline Cucurbita ficifolia (C) & - & - & - & - & - & - & - & - & 1.0 & (HR) \\
\hline Cucurbita moschata (Pumpkin) (A) & 121.3 & 51.3 & 108.7 & 59.3 & 51.3 & 219.3 & 0.44 & $5 / 4(H S)$ & - & - \\
\hline Cucurbita moschata (Pumpkin) (B) & 131.3 & 35.0 & 51.3 & 68.0 & 53.3 & 174.3 & 0.35 & 4/4 (S) & 25.0 & $(\mathrm{HR})$ \\
\hline Cucurbita moschata (Pumpkin) (C) & - & - & - & - & - & - & - & - & 1.0 & $(\mathrm{HR})$ \\
\hline Cucurbita maxima (winter squash) (A) & 57.0 & 51.0 & 13.0 & 30.0 & 20.0 & 94.0 & 0.19 & 4/4 (S) & - & - \\
\hline Cucurbita maxima (winter squash) (B) & 90.0 & 17.0 & 20.0 & 30.0 & 22.0 & 67.0 & 0.13 & 4/4 (S) & 28.3 & (R) \\
\hline Cucurbita maxima (winter squash) (C) & - & - & - & - & - & - & - & - & 1.0 & (HR) \\
\hline Lagenaria sicenaria (A) & 98.0 & 15.0 & 58.7 & 42.0 & 38.7 & 115.7 & 0.23 & $4 / 4(S)$ & - & - \\
\hline Lagenaria sicenaria (B) & 344.0 & 51.3 & 295.0 & 95.0 & 92.0 & 441.3 & 0.88 & $5 / 5$ (HS) & 65.3 & (S) \\
\hline Lagenaria sicenaria (C) & - & - & - & - & - & - & - & - & 1.0 & $(H R)$ \\
\hline Lagenaria sicenaria var Calabsha (A) & 58.3 & 15.0 & 51.7 & 42.3 & 27.3 & 109.0 & 0.21 & 4/4 (S) & - & - \\
\hline Lagenaria sicenaria var Calabsha (B) & 72.0 & 17.0 & 20.0 & 42.0 & 42.0 & 75.0 & 0.15 & $4 / 4(S)$ & 10.7 & $(\mathrm{HR})$ \\
\hline Lagenaria sicenaria var Calabsha (C) & - & - & - & - & - & - & - & - & 1.0 & $(\mathrm{HR})$ \\
\hline Luffa acutangula (A) & 112.0 & 15.0 & 30.0 & 60.0 & 50.0 & 105.0 & 0.20 & $5 / 4(H S)$ & - & - \\
\hline Luffa acutangula (b) & 226.0 & 17.0 & 35.0 & 130.0 & 110.3 & 182.0 & 0.36 & $5 / 5$ (HS) & 1.0 & $(\mathrm{HR})$ \\
\hline Luffa acutangula (C) & - & - & - & - & - & - & - & - & 1.0 & (HR) \\
\hline Luffa aegyptiaca (A) & 47.3 & 55.7 & 15.0 & 26.7 & 17.0 & 97.4 & 0.19 & 4/3 (S) & - & - \\
\hline Luffa aegyptiaca (B) & 90.0 & 15.0 & 25.0 & 40.0 & 40.0 & 80.0 & 0.18 & 4/4 (S) & 9.0 & $(H R)$ \\
\hline Luffa aegyptiaca (C) & - & - & - & - & - & - & - & - & 1.0 & $(\mathrm{HR})$ \\
\hline Benincasa hispida (A) & 21.6 & 10.0 & 17.7 & 27.2 & 24.0 & 54.8 & 0.11 & $3 / 3(\mathrm{MR})$ & - & - \\
\hline Benincasa hispida (B) & 26.6 & 17.0 & 25.3 & 37.2 & 31.0 & 79.5 & 0.16 & $3 / 4(S)$ & 1.0 & $(H R)$ \\
\hline Benincasa hispida (C) & - & - & - & - & - & - & - & - & 1.0 & $(H R)$ \\
\hline $\begin{array}{l}\text { Cucurbita maxima } x \text { C. moschata } \\
\text { (Hybrid 6001) }(\mathrm{A})\end{array}$ & 16.0 & 10.0 & 13.7 & 12.0 & 8.7 & 35.7 & 0.07 & $3 / 2$ (MR) & - & - \\
\hline $\begin{array}{l}\text { Cucurbita maxima } x \text { C. moschata } \\
\text { (Hybrid 6001) (B) }\end{array}$ & 30.0 & 17.0 & 20.0 & 17.0 & 10.3 & 54.0 & 0.11 & $3 / 2$ (MR) & 1.0 & $(H R)$ \\
\hline $\begin{array}{l}\text { Cucurbita maxima x C. moschata } \\
\text { (Hybrid 6001) (C) }\end{array}$ & - & - & - & - & - & - & - & - & 1.0 & $(H R)$ \\
\hline $\begin{array}{l}\text { Cucurbita maxima } \\
\text { Charmatoza) (A) }\end{array}$ & 57.0 & 10.0 & 7.7 & 25.0 & 15.0 & 42.0 & 0.10 & $4 / 4(S)$ & & - \\
\hline $\begin{array}{l}\text { Cucurbita maxima } \quad \text { (Hybrid } \\
\text { Charmatoza) (B) }\end{array}$ & 51.0 & 17.0 & 15.0 & 19.0 & 12.0 & 51.0 & 0.10 & $4 / 3(S)$ & 9.0 & (HR) \\
\hline $\begin{array}{ll}\text { Cucurbita maxima } & \text { (Hybrid } \\
\text { Charmatoza) }(C) & \end{array}$ & - & - & - & - & - & - & - & - & 1.0 & $(H R)$ \\
\hline Cucurbita maxima (A) & 50.0 & 27.0 & 40.0 & 32.0 & 29.3 & 99.0 & 0.19 & 4/3 (S) & - & - \\
\hline Cucurbita maxima (B) & 60.0 & 26.7 & 50.0 & 44.7 & 39.3 & 121.4 & 0.24 & 4/4 (S) & 17.0 & $(\mathrm{HR})$ \\
\hline Cucurbita maxima (C) & - & - & - & - & - & - & - & - & 1.0 & $(H R)$ \\
\hline Cucumis sativus var. Hesham (A) & 211.0 & 1180.0 & 40.0 & 100.0 & 94.0 & 1320.0 & 2.64 & $5 / 4(H S)$ & - & - \\
\hline Cucumis sativus var. Hesham (B) & 306.0 & 1295.3 & 81.0 & 181.0 & 105.0 & 1559.3 & 3.12 & $5 / 5$ (HS) & 82.3 & (HS) \\
\hline Cucumis sativus var. Hesham (C) & - & - & - & - & - & - & - & - & 58.3 & $(\mathrm{~S})$ \\
\hline New L.S.D. (5\%) & 24.4 & 6.4 & 10.6 & 15.0 & 14.6 & - & - & - & - & - \\
\hline
\end{tabular}

$A=$ Nematodes Infected Plants, $B=$ Nematodes and fungi infected plants, $C=$ fungi infected plants Scales expressed by letter(S) in parenthesis are plant response to $M$. incognita infestation according to Taylor and Sasser 1978

$* \quad \mathrm{RF}=$ Reproductive factors Nematodes final population Nematodes initial population

** $\quad$ GI/EI $=$ galls and egg-masses index according to Taylor and Sasser 1978

$* * *$ Disease symptoms index according to Kesevan and Chounhury, (1977)

$(0)=$ Immune $(1),(1)=$ Highly Resistant (HR), $(2)=$ Resistant (R), $(3)=$ Susceptible $(S),(4)=$ Highly Susceptible (HS). 
1570 EVALUATION OF SOME CUCURBITACEOUS ROOTSTOCKS ${ }^{1-}$ FOR RESISTANCE / SUSCEPTIBILITY TO ROOT- KNOT NEMATODE AND FUSARIUM WILT UNDER SCREEN-HOUSE CONDITIONS

Table 5. Different parameters of some cucurbit rootstocks infected with Meloidogyne incognita or/and Fusarium oxysporum under Screen-house condition in spring season.

\begin{tabular}{|c|c|c|c|c|}
\hline Treatments & $\begin{array}{l}\text { Root } \\
\text { Length } \\
(\mathrm{cm})\end{array}$ & $\begin{array}{l}\text { Root } \\
\text { weight (g) }\end{array}$ & $\begin{array}{c}\text { Shoot } \\
\text { Length }(\mathrm{cm})\end{array}$ & $\begin{array}{l}\text { Shoot } \\
\text { weight }(g)\end{array}$ \\
\hline Cucurbita ficifolia (A) & 23.50 & 15.9 & 37.13 & 29.36 \\
\hline Cucurbita ficifolia (B) & 28.66 & 12.50 & 39.00 & 31.80 \\
\hline Cucurbita ficifolia (C) & 12.66 & 4.13 & 20.33 & 11.86 \\
\hline Cucurbita ficifolia (D) & 33.83 & 18.53 & 37.60 & 39.03 \\
\hline Cucurbita moschata (Pumpkin) (A) & 21.66 & 12.43 & 36.30 & 22.13 \\
\hline Cucurbita moschata (Pumpkin) (B) & 25.66 & 8.46 & 37.66 & 31.96 \\
\hline Cucurbita moschata (Pumpkin) (C) & 18.66 & 6.33 & 20.33 & 17.33 \\
\hline Cucurbita moschata (Pumpkin) (D) & 29.33 & 14.6 & 39.03 & 30.43 \\
\hline Cucurbita maxima (winter squash) (A) & 17.53 & 26.63 & 33.26 & 22.53 \\
\hline Cucurbita maxima (winter squash) (B) & 19.00 & 21.60 & 33.93 & 24.77 \\
\hline Cucurbita maxima (winter squash) (C) & 16.33 & 20.00 & 27.73 & 21.80 \\
\hline Cucurbita maxima (winter squash) (D) & 21.06 & 21.50 & 39.20 & 27.96 \\
\hline Lagenaria sicenaria $(\mathrm{A})$ & 14.83 & 8.00 & 21.00 & 15.33 \\
\hline Lagenaria sicenaria (B) & 15.33 & 6.10 & 25.00 & 21.96 \\
\hline Lagenaria sicenaria (C) & 20.00 & 12.83 & 16.66 & 15.46 \\
\hline Lagenaria sicenaria (D) & 21.13 & 15.20 & 110.13 & 28.83 \\
\hline Lagenaria sicenaria var Calabsha $(\mathrm{A})$ & 21.23 & 12.86 & 33.10 & 16.63 \\
\hline Lagenaria sicenaria var Calabsha (B) & 25.23 & 10.33 & 39.66 & 23.53 \\
\hline Lagenaria sicenaria var Calabsha (C) & 19.00 & 14.76 & 20.66 & 16.73 \\
\hline Lagenaria sicenaria var Calabsha (D) & 28.40 & 16.86 & 42.50 & 30.06 \\
\hline Luffa acutangula (A) & 23.33 & 10.73 & 48.80 & 22.13 \\
\hline Luffa acutangula (B) & 25.66 & 9.03 & 62.06 & 23.56 \\
\hline Luffa acutangula (C) & 15.66 & 9.73 & 72.33 & 15.40 \\
\hline Luffa acutangula (D) & 29.56 & 15.33 & 63.36 & 24.53 \\
\hline Luffa aegyptiaca $(\mathrm{A})$ & 27.20 & 15.80 & 94.23 & 22.50 \\
\hline Luffa aegyptiaca (B) & 34.83 & 13.00 & 139.66 & 29.03 \\
\hline Luffa aegyptiaca (C) & 19.66 & 10.16 & 78.40 & 18.46 \\
\hline Luffa aegyptiaca (D) & 44.73 & 18.90 & 141.33 & 34.30 \\
\hline Benincasa hispida (A) & 22.66 & 4.36 & 42.00 & 31.00 \\
\hline Benincasa hispida $(\mathrm{B})$ & 20.30 & 2.86 & 35.73 & 26.80 \\
\hline Benincasa hispida (C) & 22.00 & 9.06 & 29.36 & 18.76 \\
\hline Benincasa hispida (D) & 20.60 & 10.76 & 35.76 & 19.26 \\
\hline $\begin{array}{l}\text { Cucurbita maxima x C. moschata (Hybrid } \\
6001)(\mathrm{A})\end{array}$ & 20.03 & 18.23 & 33.66 & 19.60 \\
\hline $\begin{array}{l}\text { Cucurbita maxima x C. moschata (Hybrid } \\
6001)(B)\end{array}$ & 21.66 & 15.03 & 35.90 & 22.87 \\
\hline $\begin{array}{l}\text { Cucurbita maxima x C. moschata (Hybrid } \\
6001)(C)\end{array}$ & 27.00 & 9.36 & 23.33 & 13.16 \\
\hline $\begin{array}{l}\text { Cucurbita maxima } x \text { C. moschata (Hybrid } \\
6001)(D)\end{array}$ & 28.86 & 15.96 & 42.16 & 29.46 \\
\hline $\begin{array}{l}\text { Cucurbita maxima (Hybrid Charmatoza) } \\
\text { (A) }\end{array}$ & 28.30 & 16.73 & 29.83 & 20.40 \\
\hline $\begin{array}{l}\text { Cucurbita maxima (Hybrid Charmatoza) } \\
\text { (B) }\end{array}$ & 32.66 & 16.20 & 34.93 & 25.93 \\
\hline $\begin{array}{l}\text { Cucurbita maxima (Hybrid Charmatoza) } \\
\text { (C) }\end{array}$ & 18.33 & 13.43 & 20.66 & 13.66 \\
\hline $\begin{array}{l}\text { Cucurbita maxima (Hybrid Charmatoza) } \\
\text { (D) }\end{array}$ & 35.46 & 19.10 & 38.90 & 32.50 \\
\hline Cucurbita maxima $(\mathrm{A})$ & 8.36 & 37.23 & 12.33 & 10.30 \\
\hline Cucurbita maxima (B) & 10.33 & 35.40 & 21.33 & 14.23 \\
\hline Cucurbita maxima (C) & 10.66 & 13.73 & 28.66 & 5.00 \\
\hline Cucurbita maxima (D) & 12.80 & 62.76 & 33.80 & 18.23 \\
\hline Cucumis sativus var. Hesham(A) & 13.76 & 15.03 & 98.70 & 30.33 \\
\hline Cucumis sativus var. Hesham(B) & 11.93 & 10.06 & 74.76 & 28.76 \\
\hline Cucumis sativus var. Hesham (C) & 14.33 & 14.06 & 23.66 & 15.46 \\
\hline Cucumis sativus var. Hesham (D) & 16.66 & 19.63 & 120.66 & 34.00 \\
\hline New L.S.D. (5\%) & 4.80 & 3.40 & 12.90 & 6.50 \\
\hline
\end{tabular}

$A=$ Nematodes Infection plants

$\mathrm{C}=$ Fusarium infected plants

$B=$ Nematodes and fungi infected plants $\mathrm{D}=$ Non - infected plants (Control) 
Table 6. Enzyme and lignin contents of some cucurbit plants in relation to nematode and fungus infestation.

\begin{tabular}{|c|c|c|c|c|c|c|}
\hline \multirow[b]{2}{*}{ Cucurbit plants } & \multicolumn{3}{|c|}{ In spring season } & \multicolumn{3}{|c|}{ In autumn season } \\
\hline & $\begin{array}{c}\text { Polyphenol } \\
\text { oxidase } \\
\text { contents } \\
\text { mg/g }\end{array}$ & $\begin{array}{c}\text { Peroxidase } \\
\text { contents } \\
\mathrm{mg} / \mathrm{g}\end{array}$ & $\begin{array}{c}\text { Lignin } \\
\text { content } \\
\mathrm{mg} / \mathrm{g}\end{array}$ & $\begin{array}{c}\text { Polyphenol } \\
\text { oxidase } \\
\text { contents } \\
\text { mg/g }\end{array}$ & $\begin{array}{l}\text { Peroxidase } \\
\text { contents } \\
\mathrm{mg} / \mathrm{g}\end{array}$ & $\begin{array}{r}\text { Lignin } \\
\text { content } \\
\mathrm{mg} / \mathrm{g}\end{array}$ \\
\hline Cucurbita ficifolia & 0.035 & 0.745 & 0.245 & 0.032 & 0.722 & 0.234 \\
\hline $\begin{array}{l}\text { Cucurbita moschata } \\
\text { (Pumpkin) }\end{array}$ & 0.019 & 0.517 & 0.071 & 0.013 & 0.051 & 0.070 \\
\hline $\begin{array}{l}\text { Cucurbita maxima (winter } \\
\text { squash) }\end{array}$ & 0.052 & 0.772 & 0.106 & 0.063 & 0.785 & 0.110 \\
\hline Lagenaria sicenaria & 0.059 & 0.456 & 0.378 & 0.065 & 0.460 & 0.379 \\
\hline $\begin{array}{l}\text { Lagenaria sicenaria var } \\
\text { Calabsha }\end{array}$ & 0.002 & 0.610 & 0.275 & 0.001 & 0.600 & 0.274 \\
\hline Luffa acutangula & 0.014 & 1.053 & 0.122 & 0.016 & 1.049 & 0.144 \\
\hline Luffa aegyptiaca & 0.047 & 0.375 & 0.264 & 0.051 & 0.330 & 0.240 \\
\hline $\begin{array}{l}\text { Lagenaria longissima } \\
\text { (Hercules gourd) }\end{array}$ & 0.040 & 0.330 & 0.134 & 0.055 & 0.440 & 0.150 \\
\hline Benincasa hispida & 0.042 & 0.944 & 0.277 & 0.046 & 0.936 & 0.280 \\
\hline $\begin{array}{l}\text { Cucurbita maxima } \times \text { C. } \\
\text { moschata (Hybrid 6001) }\end{array}$ & 0.052 & 0.945 & 0.224 & 0.055 & 0.942 & 0.228 \\
\hline $\begin{array}{l}\text { Cucurbita maxima (Hybrid } \\
\text { Charmatoza) }\end{array}$ & 0.051 & 0.785 & 0.419 & 0.055 & 0.782 & 0.422 \\
\hline $\begin{array}{l}\text { Cucumis sativus var Hesham } \\
\text { (Cucumber) }\end{array}$ & 0.016 & 0.544 & 0.023 & 0.011 & 0.560 & 0.028 \\
\hline
\end{tabular}

\section{DISCUSSION}

The present study indicates that cucumber (Cucumis sativus var. Hesham) was ranked as susceptible and highly susceptible to $M$. incognita and $F$. oxysporum. These data are in agreement with those Oteifa and Elgindi (1982), Todd et al. (1991) and Amin (1995) who indicated that none of cucumber cultivar was found to be resistant against $M$. incognita infestation.

Data indicate that winter squash, Cucurbita maxima was rated as resistant and highly resistant when the pots inoculated with nematode only and nematode plus fungus, respectively. Lagenaria sicenaria rated as moderately resistant to root-knot nematode, Meloidogyne incognita in both cases in autumn season and resistant to Fusarium wilt fungus. While, in spring season when the pots inoculated with nematode only, Benincasa hispida and Cucurbita maxima hybrid 6001 were rated as moderate resistant to root-knot nematode, Meloidogyne incognita and resistant to Fusarium wilt fungus. Regarding the chemical response, data indicated that resistant 
and highly resistant rootstocks to both diseases recorded the highest average of Polyphenol oxidase, Peroxidase and Lignin in most cases. These results are conformable to their resistant to infestation with nematode and fungus. These results are agree with results of Owens and Novothy (1966) who found that plant tissues infested by nematodes showed a considerable increases in the levels of free amino acids, organic acids and protein, also with Zhang et al.(2010) who concluded that phenylpropane metabolites had close correlation with resistance to Southern root knot nematodes in grafted cucumber seedlings. Increase in the contents of such components responsible for synthesis of auxins, hormones and many other compounds are involved in the defense mechanisms of plant to nematodes and fungi diseases many plants respond to pathogens by eliciting a hypersensitive reaction of the site of infection and subsequently, a systemic resistance response develops (Sirohi and Dasgupta, 1993). It was reported that resistant cultivars infected by different pathogens activate oxidative enzymes mainly peroxidase and polyphenol oxidase whose oxidation products such as quinines are toxic to the invading microorganisms (Rajasekhar et. al., 1997). Also, Mohamed et. al. (1999) showed that the contents of the enzymes, peroxidase and polyphenol oxidase increased within infected roots indicating consistency with the cultivar reaction to the root-knot nematode. Peroxidases have been reported to oxidise phenols and consequently have been assigned a role analogous to that proposed for polyphenol oxidases in the necrotic reaction (Sodium and Mohmood, 1992 and Zacheo et. al., 1993).Peroxides act a role in four physiological processes, auxin catabolism, and defense mechanism against pathogens, some respiratory processes and synthesis of lignin (Siddiqui and Mahmood, 1992) .At the same trend, Osman et al., (2012) showed that Rotylenchulus rveniformis as incompatible pathogen to potato, it increased the content of peroxidase, polyphenol oxidase and chitinase in the inoculated plants as indicator of inducing resistance to $M$. incognita. Because of this fact, the use of grafting onto resistant cucurbit rootstocks has proved to be effective for control of southern rootknot nematode, M. incognita and fusarium wilt fungus, F. oxysporium. These studies wilt be helpful in selection of cucurbit rootstocks for the development of cultivars resistant against both diseases. (Oda, 1999). 
AMIN, A.W. et. al., 
1574 EVALUATION OF SOME CUCURBITACEOUS ROOTSTOCKS ${ }^{1-}$ FOR RESISTANCE / SUSCEPTIBILITY TO ROOT- KNOT NEMATODE AND FUSARIUM WILT UNDER SCREEN-HOUSE CONDITIONS 


\section{REFERENCES}

1. Allam A. L. and J. P. Hollis. 1972. Sulfide inhibition of oxidase in rice roots. Phytopathology. 62: 634-639.

2. Amin A. W. 1995. Ecological and Biological studies for the control of root-knot nematodes, Meloidogyne species in Hungary, Ph. D Thesis., Hungarian Academy of Sciences. Budapest. Hungary. 126 pp.

3. Anonymous. 1951. Methods for Estimating Lignin. The institute of Paper Chemistry, Appleton, Wisconsin, 428pp.

4. Kesevan, V. and B. Chounhury. 1977. Screening for resistance to fusarium wilt of tomato. Sabrao Journal, 9: 57-65.

5. Maxwell, D. P. and D. F. Bateman. 1967. Changes in the activity of some oxidase in extracts of Rhizoctonia infected bean hypocotyls in relation to lesion maturation. Phytopathology, 57: 132-136.

6. Mohamed, M.A., M.M.A. Youssef AND M.M. Abd-elgawad. 1999. Measuring reaction of tomato cultivars to Meloidogyne incognita through plant appearance and enzyme activity. Int. J. Nematol.9:174-180.

7. Oda, M. 1999. Grafting of vegetables to improve greenhouse production. Extension Bulletin Food and Fertilizer Technology Center. No. 480, 11pp.

8. Osman,H.A., M.M.A. youssef, A.Y. El-Ginidi, H.H. Ameen, N.A. Abd-Elbary and A.M.S. Lashein. 2012. Effect of reniform nematode, Rotylenchulus reniformis as biotic inducer of resistance against root-knot nematode, Meloidogyne incognita in potato. J.Plant Prot.Res., 52:320-323.

9. Oteifa, B. A. and D. M. Elgindi.1982. Relative susceptibility of certain commercially important cultivars to existing biotype of Meloidogyne incognita and Meloidogyne Javanica in Nile-Delta Egypt. Proceeding of the Third Research and Planning Conference on Root-knot Nematodes, Meloidogyne spp. Coimbera (Portogal), September 13-17, pp 157-169.

10. Owens, R. G. and Novothy. 1966. iochemical alternation induced in host tissues by root-knot nematodes. Contrib Boyce Thonpons Inst., 23: 181-198.

11. Rajasekhar, P., A.K.Ganguly, and S.C. Swain. 1997. Quantitative changes in superoxide dismutase, catalase and peroxidase with reference to resistance in tomato to Meloidogyne incognita..Indian J.Nematol. 27: 79-85. 
12. Sharma, G.C., K, B. Rastogi, Y. R. Shukla and M. L. Khan. 1995. Reaction of cucumber varieties to root knot nematode Meloidogyne incognita. Annals of Agri. Res., 16: 33-35.

13. Siddiqui, Z.A. and J. Mahmood. 1992. Response of chichpea cultivars to Meloidogyne incognita Race 3 and their effect on peroxidase activity. Pak.J.Nematol. 10: 113-117.

14. Sirohi, A. and D.R. Dasgupta. 1993. Mechanism of resistance in cowpea to the root-knot nematode Meloidogyne incognita Race I. 1- Early induction of phenylalanine ammonia Lyase (E.C.Y.3.1.5) and Chlorogenic acid. Ind J.Nematol. 23:31-41.

15. Taylor, A. L. and J. N. Sasser, 1978. Biology, Identification and Control of Rootknot Nematodes, Meloidogyne species, Coop. Pub. Dept. Plant Pathol., North Carolina State Unive., and U.S. Agency, Int. Dev., Releigh NC., 111pp.

16. Todd, C. W., S. A. Walter and K. R. Barker. 1991. Resistance to root-knot nematodes in cucumber and horned cucumber. Supplement to J Nematol. 23(4S): 611-614.

17. Waller, W. and G. Duncan. 1969. Statistical Methods for Research Workers. Oliver and Boyd, London. 89pp.

18. Zacheo, G., Orlando, C. and Bleve-Zacheo.1993. Characterization of anionic peroxidases in tomato isolates infected by Meloidogyne incognita. J.Nematol. 25: 249-256.

19. Zhang, T., Liu, Shi-q., Zhang, Z., Yang Xiao, J., Ma. L., Huang Z. and K. Chen. 2010. Relationship between Phenylpropanes metabolism and grafted cucumber resistance to southern root-knot nematode. College of Horticulture Science and Engineering. Accepted online, 2010-8-24, (22): 54-58. 


\section{تقييم بعض الاصول من نباتات الفصيلة القرعية للإصابة}

\section{بنيماتودا تعقد الجذور والذبول الفيوزارمى تحت ظروف الصوية السلكية}

\section{امين وفدى امين1 منى عبد الونيس محمد2 تماضر عبد الرحمن جمعة3}

$$
1 \text { 1. قسم الحيوان والنبياتودا الزراعى - جامعة القاهة- كلبة الزراعة . }
$$

2. شعبة بحوث الخضر والنباتات الطبية والعطرية - مركز البحوث الزراعية - معهد بحوث البساتين

$$
\text { 3. معز بحوث الامراض - مركز البحوث الزراعية . }
$$

تعتبر نيمـاتودا تعقد الجذور ميليودوجين أنكوجنيتـا واللذبول الفيوزريـومى المتسـبب عن الفطر

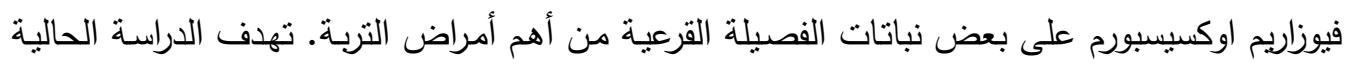

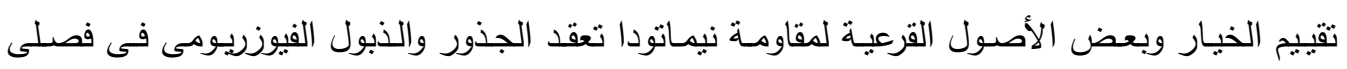
الخريبف والربيع 2009 - 2010 تحت ظروف الصوبة السلكية. تشير البيانات بأن الخيار Cucumis sativus حساس وشديد الحساسية لنيماتودا تعقد الجذور

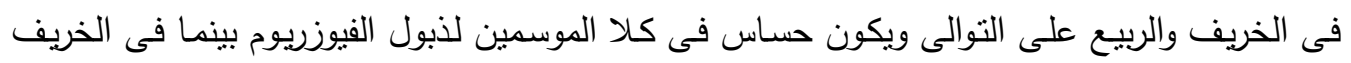
كانت الكوسة الثتوى winter squash, Cucurbita maxima أصل عالى المقاومة ومقاوم للنيمانودا عند زراعتها فى تربـة محقونه بالنيماتودا فقط أو بالنيماتودا والفيوزاريوم معاً على التوالى فالى كما أعطت

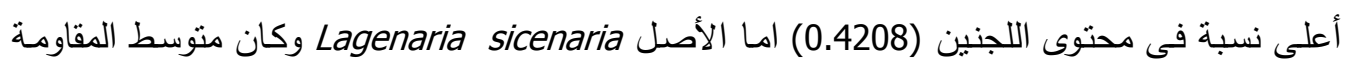

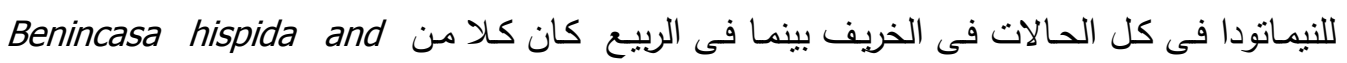
متوسط المقاومة لنيماتودا تعقد الجذور . Cucurbita maxima hybrid 6001 معظم الأصول القرعية مقاومة وعالية المقاومة للذبول الفيوزيومى فى حين الخيار كأصل اعتبر حساس وعالى الحساسية عند نمو الثتلات فى تربـة محقونـة بالفيوزاريوم فقط أو بالفيوزاريم والنيماتودا

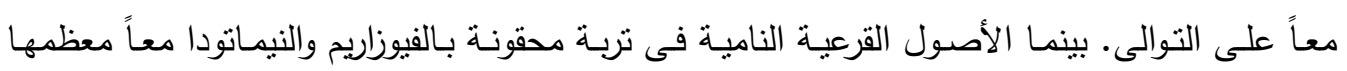

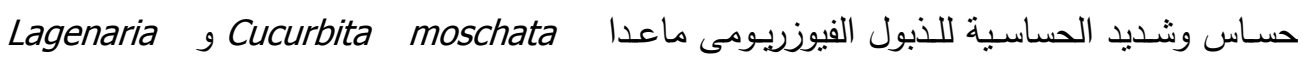

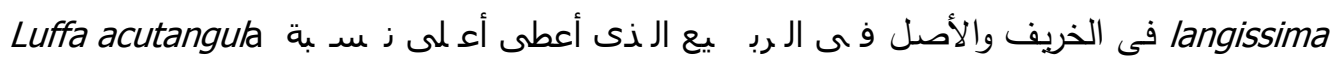

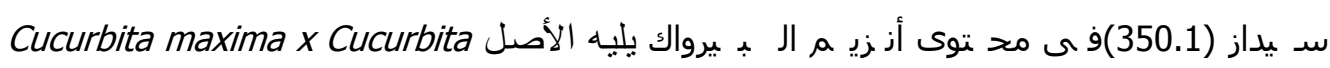
- (0.985)Hybrid Charmatoza

كما أوضحت النتائج حدوث انخفاض فى الطول والوزن الطازج للمجموع الخضرى والمجموع

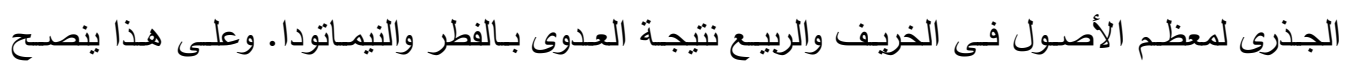

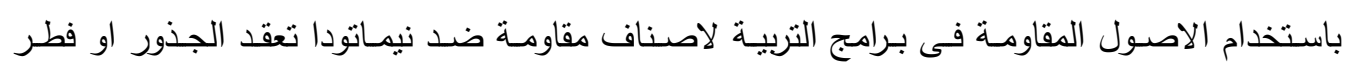
الذبول. 